\title{
Screening and identification of the differentially expressed proteins in neonatal rat kidney after partial unilateral ureteral obstruction
}

\author{
QI ZHAO $^{1}$, YANSHENG XUE ${ }^{2}$, YI YANG $^{1}$, ZHIBIN NIU $^{1}$, CHANGLIN WANG $^{1}$, \\ YING HOU $^{1}$ and HUI CHEN ${ }^{1}$
}

\author{
${ }^{1}$ Department of Pediatric Surgery, Shengjing Hospital of China Medical University, Shenyang, Liaoning 110004; \\ ${ }^{2}$ Department of Pediatric Surgery, Hongqi Hospital Affiliated to Mudanjiang Medical University, \\ Mudanjiang, Heilongjiang 157011, P.R. China
}

Received May 26, 2015; Accepted May 4, 2016

DOI: $10.3892 / \mathrm{mmr} .2016 .5338$

\begin{abstract}
Renal fibrosis, considered to be a common consequence of progressive renal disease, involves glomerulosclerosis and/or tubulointerstitial fibrosis. Currently, research is focused on investigating potential mechanisms to prevent or reverse the damage caused by fibrosis. Under the influence of cytokines, chemokines and other signaling molecules, the cellular interactions that regulate the development of interstitial fibrosis are complex. Epithelial-mesenchymal transition (EMT) has emerged as an important pathway leading to the generation of matrix-producing fibroblasts and myofibroblasts in diseased kidneys. The proteomics study compared the protein profiles between the time points of podocyte EMT and tubular cell EMT in a partial unilateral ureteral obstruction (PUUO) model in rats. Proteins isolated from the PUUO group and corresponding sham rat kidney tissues were subjected to 2-D gel electrophoresis and were then identified by mass spectrometry. In total, 43 proteins with differential expression were identified, which were reported to be involved in the regulation of the cytoskeleton and actin, glucose metabolism, cell apoptosis, mitochondrial energy metabolism, oxidative stress and endoplasmic reticulum stress. Electron transfer flavoprotein, $\beta$ polypeptide was detected by immunoblot analysis and its mRNA levels were determined in renal tissues. The results demonstrate protein alterations that reflect the pathology of the obstructed kidneys, and thus may aid in understanding the pathogenesis of obstructive nephropathy.
\end{abstract}

Correspondence to: Dr Yi Yang, Department of Pediatric Surgery, Shengjing Hospital of China Medical University, 36 Sanhao Street, Heping, Shenyang, Liaoning 110004, P.R. China

E-mail: yangy2@sj-hospital.org

Key words: proteome, neonatal, obstructive nephropathy, epithelial mesenchymal transition, electron transfer flavoprotein, $\beta$ polypeptide

\section{Introduction}

Obstructive nephropathy is a major cause of renal failure, particularly in infants and children (1). Kidney fibrosis is an inevitable outcome of progressive obstructive nephropathy (2). Obstructive nephropathy can be congenital or acquired. Congenital obstructive nephropathy can be easily identified by prenatal ultrasonography, and the number of patients diagnosed with obstructive nephropathy has increased; the prevalence is $\sim 0.5 \%$ of fetuses, following the introduction of prenatal ultrasonography as a screening method (3). Obstructive nephropathy in infants and children results primarily from the consequence of congenital renal maldevelopment, occurs during early kidney development and affects renal morphogenesis, maturation and growth (4). In the most severe cases, this renal injury will ultimately entail progressive renal tubular atrophy and inevitable interstitial fibrosis with loss of nephrons (5).

Despite intense research, the lack of a comprehensive understanding of the pathogenesis of renal scar formation following injury remains a major obstacle in developing effective therapeutic strategies. In the past several years, epithelial-mesenchymal transition (EMT), a process by which fully differentiated epithelial cells undergo transition to a fibroblast phenotype, has emerged as an important pathway leading to the generation of matrix-producing fibroblasts and myofibroblasts in diseased kidneys (5). Under the influence of cytokines, chemokines and other signaling molecules, the cellular interactions that regulate the development of interstitial fibrosis are complex (6). Proteomics studies facilitate the direct understanding of the mechanisms of physiological and pathological processes. Thus, the present study utilized 2-D electrophoresis (2-DE) gel-based proteomics to analyze the protein profiles of rat kidney tissues with experimentally induced neonatal partial unilateral ureteral obstruction (PUUO). This was conducted in order to provide insights into the pathogenesis of obstructive nephropathy and develop a network of proteins that may be targeted to prevent or reverse EMT in obstructive nephropathy. 


\section{Materials and methods}

Experimental animals and surgical procedures. This study included 26 Sprague Dawley (SD) rats (Shengjing Hospital of China Medical University Experimental Animal Center, Shenyang, China; age, 1 day; weight, 6-8 g), randomized into four groups: PUUO1d, 1 day following PUUO ( $n=6), P U U O 2 d$, 2 days following PUUO $(\mathrm{n}=8)$, PUUO5d, 5 days following PUUO $(n=6)$ and sham surgery $(n=6)$.

After general anesthesia with isoflurane inhalation, partial obstruction of the left ureter was conducted according to a modified version of the technique demonstrated by Ulm and Miller (7). Briefly, the left ureter was exposed via an abdominal transperitoneal incision using a microscope with $\mathrm{x} 4$ magnification. The underlying psoas muscle was split longitudinally to form a groove, into which the upper one-third of the left ureter was embedded. The muscle edges were closed by $8-0$ sutures. In the sham group, a laparotomy was performed and the left ureter was exposed and manipulated, but not ligated. The incision was closed in a single layer. Subsequently, the rats were placed in an animal facility with mother rats until they recovered from the anesthetic.

At different time points (1,2 and 5 days) the rats were sacrificed using an overdose of $10 \%$ chloral hydrate (Aoxin Chemical Co., Ltd., Yangzhou, China), and the left kidney was excised, decapsulated, and the kidney volume, renal pelvis and the thickness of renal parenchyma was measured immediately. Half of the kidney was snap frozen in liquid nitrogen at $-80^{\circ} \mathrm{C}$. The other half was fixed in $10 \%$ neutral-formalin, embedded in paraffin and sectioned $(4 \mu \mathrm{m})$ on a microtome for light microscopy.

No rats from the PUUO group or the sham group had a wound infection or were eaten by mother rats prior to sacrifice. The mother rats had free access to a standard rodent diet and were housed with a 12 -h light/dark cycle at $22^{\circ} \mathrm{C}$ and $55 \pm 5 \%$ relative humidity. All experiments were conducted under the Shengjing Hospital of China Medical University procedural and ethical guidelines.

Double immunofluorescence staining. Double immunofluorescence labeling for podocalyxin, ED-1, E-cadherin and $\alpha$-smooth muscle actin ( $\alpha$-SMA) was performed on paraffin-embedded kidney sections to label the podocytes, macrophages, tubular epithelial cells and myofibroblasts in the kidney. Sections $(4 \mu \mathrm{m})$ pre-treated using heat-induced antigen retrieval were stained with polyclonal rabbit anti-podocalyxin (1:50; cat. no. ab205350; Abcam, Cambridge, MA, USA), monoclonal mouse anti-ED-1 (1:50; cat. no. MAB1435; Chemicom, Toronto, ON, Canada), polyclonal rabbit anti-E-cadherin (1:50; cat. no. ab53226; Abcam) and monoclonal mouse anti- $\alpha$-SMA (1:50; cat. no. ab7817; Abcam) overnight at $4^{\circ} \mathrm{C}$. They were then incubated with fluorescein isothiocyanate and rhodamine-conjugated goat anti-mouse (cat. no. sc-3796) and goat anti-rabbit (cat. no. sc-3839) secondary antibodies at 1:100 (Santa Cruz Biotechnology, Inc., Santa Cruz, CA, USA). Negative control was performed by replacing the primary antibody with phosphate-buffered saline. To stain the nuclei, 4',6-diamidino-2-phenylindole (Bioss, Inc., Beijing, China) was used. The sections were examined by a fluorescence microscope (Nikon CE1 Confocal Microscope, Nikon, Tokyo,
Japan). Renal tissues with positive double immunofluorescence staining were used for future 2-DE. Negative tissues were abandoned.

2-DE screening. For 2-DE, the proteins from 6 kidney tissues from each experimental group were pooled and a set of 12 gels were run, with one pooled sample run in triplicate per group for reproducibility. The tissue proteins were prepared with an acetone precipitation method (8) and were quantified using the 2D-Quant kit (GE Healthcare, Little Chalfont, UK). Isoelectrofocusing (IEF) and sodium dodecyl sulfate-polyacrylamide gel electrophoresis (SDS-PAGE) were performed according to the manufacturer's instructions of the Ettan IPGphor IEF system (GE Healthcare). Specifically, $900 \mu \mathrm{g}$ total protein was mixed with hydration solution to a total volume of $450 \mu$ l. Hydration and IEF electrophoresis were automatically processed on the electrode plate of the IPGphor horizontal electrophoresis system at $20^{\circ} \mathrm{C}$. A homogeneous $12.5 \%$ gel $(0.75 \mathrm{~mm})$ was used for the second phase vertical SDS-PAGE gel electrophoresis. After electrophoresis, the gel was stained with modified colloidal Coomassie Brilliant Blue (mcCBB) G-250 as described previously (8).

Image acquisition and data analysis. The gels were scanned with a Power Look 2100XL image scanner (Umax, Taipei, Taiwan). Spot detection, quantification and matching involved the use of 2-D gel analysis software (Image Master 2-D platinum 6.0, GE Healthcare) with the CBB-stained gels. The relative volume of spots [\% volume calculated as the spot volume (the sum of the intensities of the pixel units within the protein spot) normalized as a percentage of the total volume of all the spots present in a gel] was obtained from 3 parallel experiments. Spots with at least 2 -fold difference in $\%$ volume, indicated to be statistically significant $(\mathrm{P}<0.05)$, were defined as differentially expressed proteins and were excised for further analysis.

In-gel digestion and matrix-assisted laser desorption/ionization time-of-flight mass spectrometry (MALDI-TOF MS). Spots were selected manually and destained in 50\% acetonitrile (ACN) in $25 \mathrm{mM}$ ammonium bicarbonate buffer, dried in the SpeedVac and re-hydrated in trypsin solution $(15 \mu \mathrm{g} / \mathrm{ml})$ for $1 \mathrm{~h}$ at $4^{\circ} \mathrm{C}$, followed by the addition of $5 \mathrm{ml} 25 \mathrm{mM}$ ammonium bicarbonate buffer to immerse the gel fragments. After incubation for $16 \mathrm{~h}$ at $37^{\circ} \mathrm{C}$, the digested peptides were extracted with $5 \%$ trifluoroacetic acid (TFA) and $2.5 \% \mathrm{TFA} / 50 \% \mathrm{ACN}$ at $37^{\circ} \mathrm{C}$ for $1 \mathrm{~h}$ separately. Tryptic peptides were finally dissolved in MALDI matrix $(5 \mathrm{mg} / \mathrm{ml} \alpha$-cyana-4-hydroxycinnamic acid in $0.1 \%$ TFA and 50\% ACN), and spotted onto 192-well stainless steel MALDI target plates (8). The sample plate was placed into a MALDI-TOF mass spectrometer (Applied Biosystems, Waltham, MA, USA) for mass spectrometry to obtain peptide mass fingerprinting. The MS and MS/MS spectra were subsequently searched against the IPI.RAT. v3.83 database (http://www.ebi.ac.uk/IPI), with use of GPS (version 3.0; Applied Biosystems) and MASCOT (version 2.0; Matrix Science, London, UK) database search algorithms. The following search criteria were used: Trypsin specificity, cysteine carbamidomethylation and methionine oxidation as variable modifications, 2 trypsin miscleavage allowed, $0.2 \mathrm{Da}$ 
MS tolerance and 0.3 Da MS/MS tolerance. Protein identifications were accepted with a Mowse score (9) $\geq 23$ and $\mathrm{P}<0.05$. Differentially expressed proteins were investigated using Gene Ontology (http://www.geneontology.org) for molecular function, biological processes and cellular components.

Immunoblot analysis. Protein was isolated using the isolation kit from Beyotime Institute of Biotechnology (Haimen, China) according to the manufacturer's instructions, and was quantified using the 2D-Quant kit. In total, $50 \mu \mathrm{g}$ protein extract from rats was separated by $12 \%$ SDS-PAGE and then transferred with Tris-HCl methanol (20 mM Tris, $150 \mathrm{mM}$ glycine and $20 \%$ methanol) onto polyvinylidene difluoride membranes (EMD Millipore, Billerica, MA, USA) in a trans-blot electrophoresis transfer cell (Bio-Rad, Hercules, CA, USA). Membranes were probed with antibodies against electron transfer flavoprotein, $\beta$ polypeptide (ETFB; polyclonal goat anti-rat; 1:250; cat. no. ab104944; Abcam Ltd., Hong Kong, China) or actin (1:2,000; polyclonal goat anti-rat; cat. no. sc-1616; Santa Cruz Biotechnology, Inc.). All immunoblots were run at least in triplicate. Visualization of the antigen-antibody complexes was conducted using enhanced chemiluminescence reagents (Pierce Biotechnology, Rockford, IL, USA). Detected bands were quantified by Image $2 \mathrm{x}$ software (version 2.1.4.7; National Institutes of Health, Bethesda, MA, USA). The relative density of each protein was calculated by dividing the optical density value of each protein by that of the loading control.

Reverse transcription-quantitative polymerase chain reaction (RT-qPCR). Total RNA was extracted using TRIzol reagent (Invitrogen; Thermo Fisher Scientific Inc., Waltham, MA, USA), according to the manufacturer's instructions. cDNA was synthesized from $3 \mu \mathrm{g}$ RNA using the Takara RNA PCR kit (Takara, Bio Inc., Otsu, Japan). qPCR amplifications were performed in triplicate on a Light Cycler (Roche Applied Science, Penzberg, Germany) with the following primers: Sense: 5'ATGAGCCTC GCTATGCCACAC3' and antisense: 5'ACCTTGGAGGTC AGGTCCACAC3' for ETFB; and sense: 5'GGAGATTACTGC CCTGGCTCCTA3' and antisense: 5'GACTCATCGTACTCC TGCTTGCTG3' for $\beta$-actin. The house keeping gene $\beta$-actin was used as an endogenous control. The amplification profile involved $5 \mathrm{sec}$ of denaturation at $95^{\circ} \mathrm{C}$ and $20 \mathrm{sec}$ of annealing at $64^{\circ} \mathrm{C}$ for 45 cycles. The relative mRNA levels for each sample were calculated using the $2^{-\Delta \Delta \mathrm{Cq}}$ method (10).

Statistical analysis. All statistical analyses were performed using SPSS version 18.0 (SPSS, Inc., Chicago, IL, USA). Data are expressed as the mean \pm standard deviation (\% volume of spots for 2-DE analysis, and relative density of bands on immunoblot analysis). Two-tailed Student's t-test was employed for analyzing significant differences compared with the sham group. A normality test and homogeneity of variance from the PUUO and sham group was analyzed using two-way analysis of variance. $\mathrm{P}<0.05$ was considered to indicate a statistically significant difference.

\section{Results}

Morphological changes of the obstructed kidney. By gross anatomy, the appearance of rat kidney in the PUUO1d group was not enlarged, and the renal pelvis was not dilated. By gross anatomy, there was no visible hydronephrosis. In the PUUO2d group, the hyperemia kidney appeared as increased kidney volume, visible hydronephrosis and dilated renal pelvis. The damage was increasingly severe with the persistence of the obstruction and hydronephrosis, and a thin cortex were observed in the kidneys from the PUUO5d group. The kidneys from rats in the of sham surgery group showed normal morphology.

Double immunofluorescence staining. Podocalyxin is the hallmark of podocytes, while ED-1 is the hallmark of macrophages. As presented in Fig. 1, positive double labeling for podocalyxin and ED-1 on day 2 after obstruction (Fig. 1B) revealed that podocytes took on mesenchymal characteristics. Similarly, E cadherin is the marker of tubular epithelial cell, $\alpha$-SMA is the marker of myofibroblasts, and the positive double labeling for them indicated that phenotypic changes in tubular epithelial cells occurred on day 5 after PUUO (Fig. 1C). The phenotypic changes in podocytes induced by PUUO occurred earlier compared with the tubular epithelial cells. Thus, in the PUUO2d group, kidneys from the two rats that did not exhibit phenotypic changes were not included in the rest of the experiments.

Protein profiles of rat kidney tissues from the sham surgery group and those with PUUO. The rat kidney tissue proteome contained $\sim 600$ detectable proteins on a single mcCBB-stained 2-DE gel (Fig. 2). More than $80 \%$ of the protein spots were matched on the 4 sets of CBB-stained gels. Overall, the 2-DE protein spot patterns across all the gels were similar in all groups. However, 46 protein spots with at least 2-fold difference in $\%$ volume $(\mathrm{P}<0.05)$ between kidney tissues from the sham surgery group and the PUUO groups were detected and were dissected for further analysis. Of the 46 dissected spots, 43 were also identified to be differentially expressed by MS (Table I).

Immunoblot analysis of selected proteins. ETFB was selected for further immunoblot analysis. ETFB was enhanced initially following PUUO compared with the sham group, and was then continuously downregulated with sustained obstruction. In the PUUO5d group, no significant difference was identified in ETFB levels compared with the sham group. The result is consistent with the results of $2 \mathrm{D}-\mathrm{E}(\mathrm{P}<0.05)$ (Fig. 3A-C).

Transcription levels. According to the relative mRNA levels (Fig. 3D), the mRNA expression level of ETFB was not identified to be significantly different in the PUUO groups compared with the sham group $(\mathrm{P}>0.05)$.

\section{Discussion}

Despite numerous clinical and experimental studies over the past several decades, the pathogenesis of obstructive nephropathy remains to be fully understood. Following urinary tract obstruction and tubular dilatation, a cascade of events results in cellular proliferation and tubular apoptosis, which leads to tubular atrophy and inevitably progressive fibrosis (6). EMT, which is often preceded by and closely associated with chronic 

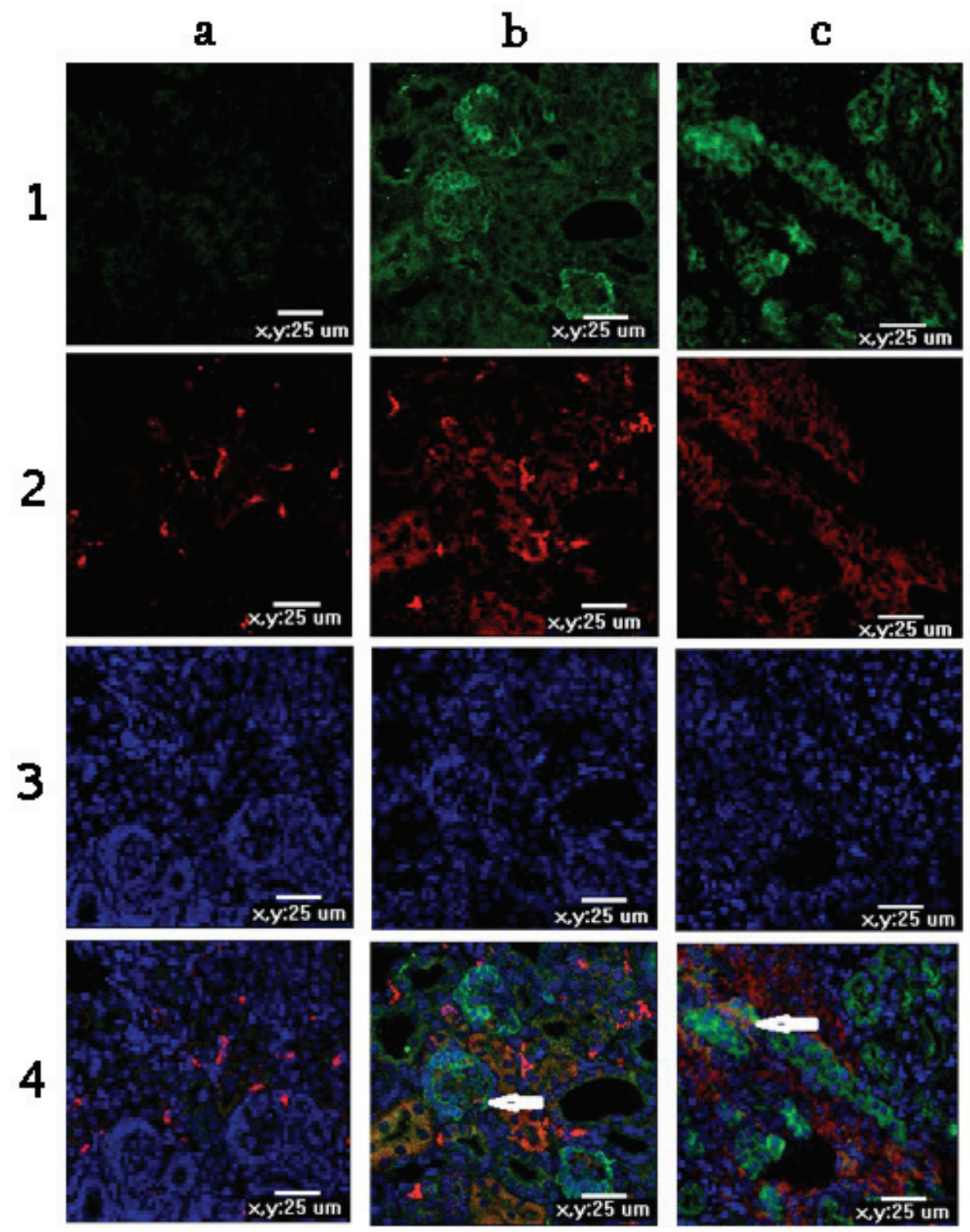

Figure 1. Double immunofluorescence staining of neonatal PUUO (magnification, x800). (A) PUUO1d: (A1) green labeling of podocalyxin in glomeruli; (A2) red labeling of ED-1; (A3) blue cell nuclei stained by DAPI; and (A4) merge. No phenotypic changes of the podocytes were observed in this group. (B) PUUO2d: (B1) green labeling of podocalyxin in glomeruli; (B2) red labeling of ED-1; (B3) blue cell nuclei stained by DAPI; (B4) merge. Podocytes exhibited mesenchymal characteristics (indicated by arrow). (C) PUUO5d: (C1) green labeling of E-cadherin in renal tubule; (C2) red labeling of $\alpha$-smooth muscle actin; (C3) blue cell nuclei stained by DAPI; (C4) merge. Phenotypic changes in tubular epithelial cells were observed (indicated by arrow). PUUO, partial unilateral ureteral obstruction; PUUO1d, 1 days following PUUO; PUUO2d, 2 days following PUUO; PUUO5d, 5 days following PUUO.

interstitial inflammation, could be an adaptive response of epithelial cells to a hostile or changing microenvironment, and it is increasingly recognized as an integral part of tissue fibrogenesis following injury (5). In addition to tubular epithelial cells, recent studies indicate that glomerular podocytes may also undergo transition following injury (9). Phenotypic alteration of podocytes results in functional impairment, resulting in proteinuria and glomerulosclerosis (9). Thus, it was hypothesized that EMT of epithelial cells and podocytes is involved in the initial phases of renal fibrosis.

Double labeling revealed that ED- 1 and $\alpha$-SMA were undetectable in the majority of glomeruli in healthy neonatal kidneys, whereas ED-1 was detected in glomeruli from the PUUO1d group and levels progressively increased after day 2 of PUUO. In addition, $\alpha$-SMA was observed in renal tubular epithelial cells and was detectable in the majority of renal tubular epithelial cells after day 5 of PUUO. Thus, podocytes took on mesenchymal characteristics 2 days after obstruction and phenotypic changes in tubular epithelial cells occurred 5 days after PUUO.

Therefore, identification of the protein expression profile of renal tissues at the time point of podocyte and tubular cell EMT aids in the determination of the pathogenesis of renal injury and progressive interstitial fibrosis, and also increased the understanding of the onset of podocyte dysfunction, proteinuria and glomerulosclerosis.

Numerous studies using radiology have demonstrated that tubular atrophy and interstitial fibrosis develop prior to significant renal pelvic dilatation (11-13). However, the present study investigated EMT pathological changes of podocytes 
Table I. Proteins with differential expression between the PUUO groups and corresponding sham rat kidney tissues identified be matrix-assisted laser desorption/ionization time-of-flight mass spectrometry.

\begin{tabular}{|c|c|c|c|c|c|}
\hline $\begin{array}{l}\text { Protein } \\
\text { name }\end{array}$ & $\begin{array}{l}\text { Accession } \\
\text { no. }\end{array}$ & $\begin{array}{l}\mathrm{Mr}(\mathrm{kDa}) / \\
\mathrm{Pi}\end{array}$ & Expression & $\begin{array}{l}\text { Mascot } \\
\text { score }\end{array}$ & Function \\
\hline Hsp90b1 & IPI00365985 & $93 / 4.72$ & $\uparrow$ & 385 & $\begin{array}{l}\text { Endoplasmic reticulum associated } \\
\text { degradation }\end{array}$ \\
\hline Sucla2 & IPI00951645 & $48 / 6.08$ & $\uparrow$ & 125 & Tricarboxylic acid cycle \\
\hline Vcp & IPI00212014 & $90 / 5.14$ & $\uparrow$ & 478 & Export of misfolded proteins \\
\hline Acy1a & IPI00464791 & $46 / 6.03$ & $\uparrow$ & 437 & Amino acid metabolism \\
\hline Gsn & IPI00923716 & $81 / 5.46$ & $\uparrow$ & 152 & Actin-modulating protein \\
\hline Hnrnpf & IPI00210357 & $46 / 5.31$ & $\uparrow$ & 121 & Processing pre-mRNAs \\
\hline Cox $5 \mathrm{a}$ & IPI00192246 & $16 / 6.08$ & $\uparrow$ & 116 & Oxidoreductase family \\
\hline RGD1565368 & IPI00769218 & $36 / 8.44$ & $\uparrow$ & 116 & Energy metabolism \\
\hline Ezr & IPI00470254 & $69 / 5.83$ & $\uparrow$ & 214 & $\begin{array}{l}\text { Connection cytoskeletal structures } \\
\text { to the plasma membrane }\end{array}$ \\
\hline Hnrnpk & IPI00780608 & $51 / 5.39$ & $\uparrow$ & 257 & Pre-mRNA-binding protein \\
\hline Tpm4 & IPI00214905 & $29 / 4.66$ & $\uparrow$ & 184 & Stabilize cytoskeleton actin filaments \\
\hline Aldh9a1 & IPI00203690 & $57 / 6.94$ & $\uparrow$ & 121 & Aldehyde dehydrogenase family \\
\hline Eef1b2 & IPI00476899 & $25 / 4.55$ & $\uparrow$ & 141 & EF-1- $\beta / E F-1-\alpha$ family \\
\hline Pepd & IPI00364304 & $56 / 5.61$ & $\uparrow$ & 100 & Collagen metabolism \\
\hline Prdx6 & IPI00231260 & $25 / 5.64$ & $\uparrow$ & 500 & Oxidoreductase family \\
\hline Dlst & IPI00551702 & $49 / 8.89$ & $\uparrow$ & 64 & Tricarboxylic acid cycle \\
\hline Park7 & IPI00212523 & $20 / 6.32$ & $\uparrow$ & 125 & Oxidoreductase family \\
\hline Actr3 & IPI00949517 & $48 / 5.61$ & $\uparrow$ & 229 & Actin polymerization and actin networks \\
\hline Rbm3 & IPI00367437 & $17 / 7.98$ & $\uparrow$ & 62 & Cold-inducible mRNA binding protein \\
\hline Psmc2 & IPI00421600 & $49 / 5.59$ & $\uparrow$ & 385 & Degradation of ubiquitinated proteins \\
\hline Erp44 & IPI00949066 & $47 / 5.14$ & $\uparrow$ & 244 & Protein folding \\
\hline Gdi2 & IPI00197568 & $47 / 6.5$ & $\uparrow$ & 140 & Regulate the GDP/GTP exchange \\
\hline Gsn & IPI00923716 & $81 / 5.46$ & $\uparrow$ & 86 & Actin-modulating protein \\
\hline Ahcy & IPI00476295 & $48 / 6.07$ & $\uparrow$ & 99 & Amino acid metabolism \\
\hline Ezr & IPI00948980 & $69 / 5.83$ & $\uparrow$ & 194 & $\begin{array}{l}\text { Connection cytoskeletal structures } \\
\text { to the plasma membrane }\end{array}$ \\
\hline Atp5b & IPI00551812 & $56 / 5.19$ & $\uparrow$ & 148 & Mitochondrial membrane ATP synthase \\
\hline RGD1309537 & IPI00564409 & $20 / 4.67$ & $\uparrow$ & 192 & Myosin regulatory subunit \\
\hline $\mathrm{Fbp} 1$ & IPI00231745 & $40 / 5.54$ & $\uparrow$ & 198 & $\begin{array}{l}\text { Rate-limiting enzyme in } \\
\text { gluconeogenesis }\end{array}$ \\
\hline Aldh1a2 & IPI00211419 & $57 / 5.58$ & $\uparrow$ & 186 & Aldehyde dehydrogenase family \\
\hline Clta & IPI00230870 & $24 / 4.43$ & $\uparrow$ & 160 & Clathrin light chain family \\
\hline Calr & IPI00191728 & $48 / 4.33$ & $\uparrow$ & 126 & Chaperone in protein folding \\
\hline Anxa5 & IPI00471889 & $36 / 4.93$ & $\uparrow$ & 440 & Anticoagulant protein \\
\hline Cct2 & IPI00366218 & $58 / 6.01$ & $\uparrow$ & 202 & Folding of actin \\
\hline $\operatorname{Prdx} 1$ & IPI00211779 & $22 / 8.27$ & $\uparrow$ & 75 & Oxidoreductase family \\
\hline Atp5b & IPI00551812 & $56 / 5.19$ & $\uparrow$ & 98 & Mitochondrial membrane ATP synthase \\
\hline Etfb & IPI00364321 & $28 / 7.6$ & $\downarrow$ & 150 & Specific electron acceptor \\
\hline Dld & IPI00365545 & $55 / 7.96$ & $\downarrow$ & 153 & Oxidoreductase family \\
\hline Tufm & IPI00371236 & $50 / 7.23$ & $\downarrow$ & 144 & Protein biosynthesis \\
\hline Ruvbl2 & IPI00364340 & $51 / 5.49$ & $\downarrow$ & 222 & $\begin{array}{l}\text { Nucleotide-binding, ATP-binding, } \\
\text { DNA repair }\end{array}$ \\
\hline LOC100360986 & IPI00559028 & $35 / 9.4$ & $\downarrow$ & 189 & $\begin{array}{l}\text { Nucleic acid binding, nucleotide } \\
\text { binding }\end{array}$ \\
\hline $\mathrm{Tf}$ & IPI00679202 & $79 / 7.14$ & $\downarrow$ & 461 & Stimulate cell proliferation \\
\hline Apoa4 & IPI00324272 & $44 / 5.12$ & $\downarrow$ & 62 & $\begin{array}{l}\text { Chylomicrons and VLDL } \\
\text { secretion and catabolism }\end{array}$ \\
\hline Aldh4a1 & IPI00921682 & $62 / 8.26$ & $\downarrow$ & 236 & Aldehyde dehydrogenase family \\
\hline
\end{tabular}

$\uparrow$, upregulation of the protein level in the PUUO groups compared with the corresponding sham rat kidney tissues; $\downarrow$, downregulation of the protein level in the PUUO groups compared with the corresponding sham rat kidney tissues. PUUO, partial unilateral ureteral obstruction. 


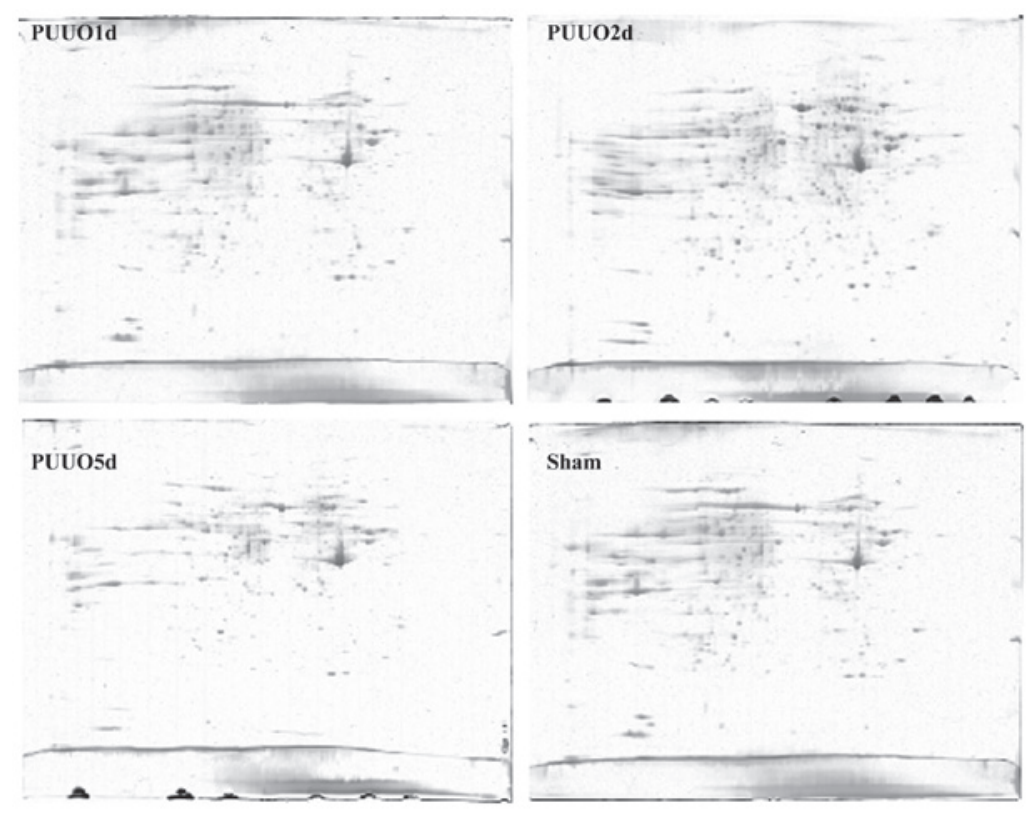

Figure 2. 2-DE of protein profiles of rat kidney tissues from the sham surgery group and the neonatal PUUO groups. Representative Coomassie-stained 2-DE gel expression maps of proteins in PUUO1d, PUUO2d, PUUO5d and sham surgery groups. 2-DE, 2-D electrophoresis; PUUO, partial unilateral ureteral obstruction; PUUO1d, 1 days following PUUO; PUUO2d, 2 days following PUUO; PUUO5d, 5 days following PUUO.

A

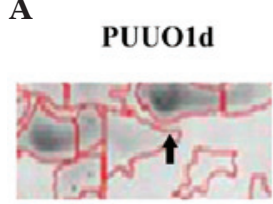

PUUO5d

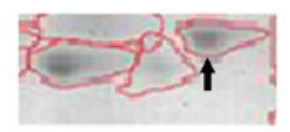

C

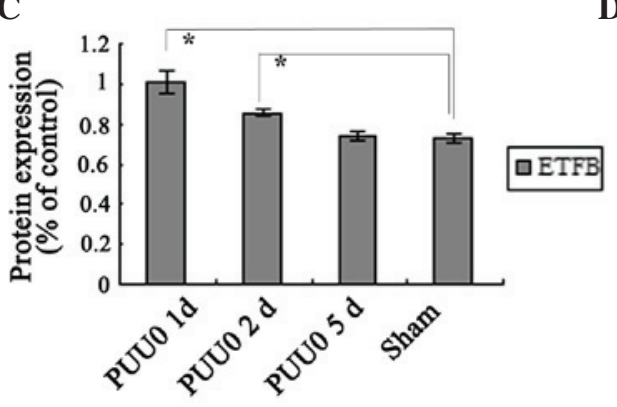

B

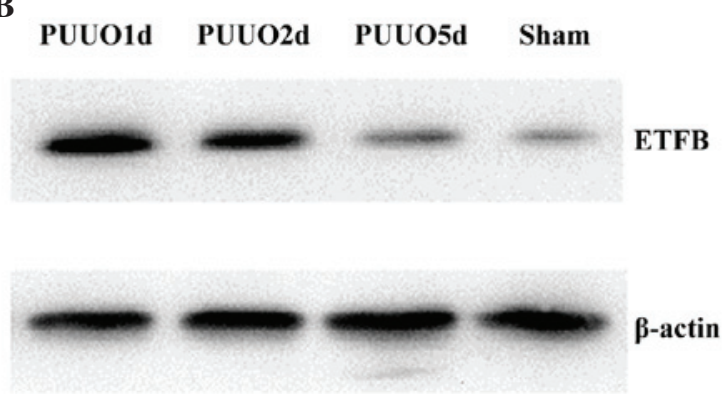

D

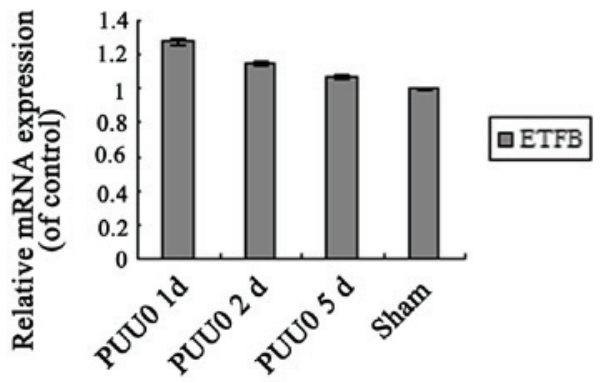

Figure 3. Immunoblot and RT-qPCR analysis of ETFB mRNA expression. (A) 2-DE gel of spot identified as ETFB (indicated by arrow). (B) Confirmation of 2-DE results by immunoblotting. Analysis of protein expression of ETFB with $\beta$-actin as the internal control. (C) Quantification of immunoblot result. Relative expression of ETFB protein. (D) Comparisons of total mRNA expression of ETFB by RT-qPCR. *P<0.05. 2-DE, 2-D electrophoresis; RT-qPCR, reverse transcription-quantitative polymerase chain reaction; PUUO, partial unilateral ureteral obstruction; PUUO1d, 1 days following PUUO; PUUO2d, 2 days following PUUO; PUUO5d, 5 days following PUUO; ETFB, electron transfer flavoprotein, $\beta$ polypeptide.

and tubular cells; determination of the differentially expressed proteins associated with EMT of podocytes and tubular cells could rule out the bias caused by different degrees of partial obstruction, and establish the diagnosis on a pathological, rather than radiological, basis. Thus, the present study used a comparative proteomics 2-DE system to observe the protein expression profile during obstructive nephropathy and a total of 43 differentially expressed proteins were successfully identified. These proteins participate in the regulation of cytoskeleton and actin, glucose metabolism, cell apoptosis, mitochondrial energy metabolism, oxidative stress and endoplasmic reticulum stress.

Results of previous studies have shown that actin is one of two monomer subunits of microfilaments, which are the 
major cytoskeleton component. In addition, actin is critical in a number of cellular functions, such as cell motility, cell division, transfer of information between cells, the maintenance of cell shape, cell connections, cell polarity and the regulation of transcription (14). Actin-regulating proteins including Gsn, Tpm4, Actr3 and Cct2 were significantly upregulated in the early stages following PUUO. GSN is a calcium-regulated actin-modulating protein, Tpm4 is implicated in stabilizing cytoskeleton actin filaments, Actr3 is involved in the regulation of actin polymerization and formation of branched actin networks, and Cet2 is known to be involved in the folding of actin in vitro (Gene Ontology). It was hypothesized that rat kidneys subjected to PUUO produce more actin filaments to control and maintain kidney shape and internal structure. However, with severe and persistent obstruction, this leads to progressive smooth muscle hyperplasia, enhanced peristalsis and finally smooth muscle fibrosis.

A total of six differentially expressed proteins were successfully identified, which participate in the regulation of mitochondrial energy metabolism. Sucla2 and Dlst are involved in the tricarboxylic acid cycle, and Acyla and Ahcy are associated with amino acid metabolism, ATP5b is involved in mitochondrial membrane ATP synthase and Tufm promotes protein biosynthesis (Gene Ontology). The expression of the majority of proteins in the kidney following obstruction were upregulated (with the exception of Tufm), suggesting that mitochondrial energy metabolism abnormalities may be the basis for early renal pathological changes in obstructive nephropathy. Due to mitochondrial dysfunction, caused by the imbalance between biogenesis and degradation, the induction of oxidative stress is induced (15), which contributes significantly to renal energy loss, redox environment damage and transforming growth factor- $\beta$ (6) pathway activation. This ultimately results in cell apoptosis and renal fibrosis.

In eukaryotic cells, newly synthesized secretory proteins enter the secretory pathway via the endoplasmic reticulum (ER). After proper folding, proteins are then transported out of the ER and folding-defective products are retained in the ER, thus initiating the ER-associated degradation process (ERAD) $(16,17)$. ER stress was demonstrated to induce EMT in human proximal tubule cells, a finding that directly connects ER stress to renal fibrosis (18). Among the differentially expressed proteins, several were clustered functionally with protein folding. Calr acts as a chaperone in promoting protein folding, oligomeric assembly and quality control in the ER via the calreticulin/calnexin cycle. Heat shock protein 90b1 is a member of the heat shock protein family, which can perform specific chaperone functions in the processing and transport of secreted proteins to ERAD. In addition, Erp44 was found to be closely associated with protein folding, response to ER stress and unfolded proteins. Psmc 2 is involved in the ATP-dependent degradation of ubiquitinated proteins and vcp is required for the export of misfolded proteins from the ER to the cytoplasm, where they are degraded by the proteasome (Gene Ontology). Thus, the elevation of these five proteins in neonatal rats with PUUO may aid in understanding the association between ER stress and obstructive nephropathy.

Among the differently expressed proteins, Prdx1, Prdx6, Park7, Dld and Cox5a, which are members of different oxidoreductase families have functions in the regulation of antioxidation related to oxidative stress. Following obstruction, increased production of intracellular reactive oxygen species (19) induces oxidative stress, leading to monocyte/macrophage (ED-1) infiltration (20) followed by apoptosis and eventually renal fibrosis. This process is regulated by pro-apoptotic and anti-apoptotic factors simultaneously (21) and involves the TGF- $\beta 1$ signaling pathway (22). Therefore, oxidative stress is vital in the regulation of inflammation, apoptosis and fibrosis.

Proteomics screening results reflected the complexity of protein expression changes in the process of renal injury. In order to confirm the proteomics results, in depth investigation was necessary. The results of 2-DE demonstrated that ETFB was enhanced initially following PUUO, and was then continuously downregulated with sustained obstruction. Gene ontology analyses mention that ETFB is a specific electron acceptor $(23,24)$ that is associated with tissue remodeling and/or fibrotic processes $(25,26)$. Thus, the present study focused on ETFB out of all the differentially expressed proteins. Immunoblot analysis and RT-qPCR were performed to investigate the differential expression of protein and mRNA between the obstructed and normal kidney. In the neonatal kidney with PUUO, immunoblot analysis confirmed that ETFB was upregulated compared with that in the sham group, which was consistent with the results of 2-DE. ETFB was continuously downregulated with persistence of the obstruction and no significant difference was identified in the expression in the PUUO5d group compared with that in the sham group. However, RT-qPCR analysis demonstrated that ETFB was not altered at the mRNA level. The inconsistency between mRNA and protein expression may be caused by posttranscriptional regulation. ETFB is located in the inner membrane of the mitochondrial matrix in a complex with ETFA, FAD and AMP, which together act as an electron acceptor of 5 isoforms of acyl-CoA dehydrogenase, glutaryl-CoA and sarcosine dehydrogenase in the fatty acid $\beta$-oxidation cascade $(23,24)$. ETFB is normally active in the mitochondria, the energy-producing centers of cells, and is involved in the process by which fats and proteins are broken down to produce energy (Gene Ontology). Based on data from Hirokawa et al $(25,26)$, ETFB participates in the mechanoregulation of fibroblast cell number and ETFB knockdown can reduce TGF- $\beta$-induced $\alpha$-SMA mRNA expression and affect tissue remodeling and/or fibrotic processes. In general, ETFB protein expression is relatively high in early obstruction and relatively low in late obstruction. Thus, its upregulation may be an attempt to promote renal fibrosis in the early stages. With continued obstruction results in mitochondrial dysfunction and kidney energy loss, ETFB may gradually decrease as it is involved in energy metabolism. Its expression was not significantly different compared with that in the sham surgery group in advanced stages.

In conclusion, an overview of proteomic changes in the kidney of neonatal rats with PUUO was demonstrated. Differentially expressed proteins caused by the primary obstruction or secondary injuries in the kidney tissues exposed to the obstructive environment were determined. These proteins possess diverse functions, such as involvement in cell cytoskeleton and energy metabolism. However, their precise role in the pathology obstructive nephropathy requires further investigation. This study may provide insights into obstruc- 
tive nephropathy and contribute to the development of novel therapeutic strategies.

\section{Acknowledgements}

This study was supported by the National Nature Science Foundation of China (grant no. 81370772) and the Nature Science Foundation of Liaoning Province, China (grant no. 2013021036).

\section{References}

1. Chevalier RL and Peters CA: Congenital urinary tract obstruction: Proceedings of the State-Of-The-Art Strategic Planning Workshop-National Institutes of Health, Bethesda, Maryland, USA, 11-12 March 2002. Pediatr Nephrol 18: 576-606, 2003.

2. Eddy AA: Molecular basis of renal fibrosis. Pediatr Nephrol 15: 290-301, 2000

3. Madsen MG, Nørregaard R, Frøkiær J and Jørgensen TM: Urinary biomarkers in prenatally diagnosed unilateral hydronephrosis. J Pediatr Urol 7: 105-112, 2011.

4. Wen JG, Ringgaard S, Jorgensen TM, Stodkilde-Jorgensen H, Djurhuus JC and Frokiaer J: Long-term effects of partial unilateral ureteral obstruction on renal hemodynamics and morphology in newborn rats: a magnetic resonance imaging study. Urol Res 30: 205-212, 2002.

5. Liu Y: New insights into epithelial-mesenchymal transition in kidney fibrosis. J Am Soc Nephrol 21: 212-222, 2010.

6. Chevalier RL: Obstructive nephropathy: Towards biomarker discovery and gene therapy. Nat Clin Pract Nephrol 2: 157-168, 2006.

7. Ulm AH and Miller F: An operation to produce experimental reversible hydronephrosis in dogs. J Urol 88: 337-341, 1962.

8. Zhao Q, Yang Y, Wang CL, Hou Y and Chen H: Screening and identification of the differential proteins in kidney with complete unilateral ureteral obstruction. Int J Clin Exp Pathol 8: 2615-2626, 2015.

9. Pappin DJ, Hojrup P and Bleasby AJ: Rapid identification of proteins by peptide-mass fingerprinting. Curr Biol 3: 327-332, 1993.

10. Livak KJ and Schmittgen TD: Analysis of relative gene expression data using real-time quantitative PCR and the 2(-Delta Delta C(T)) Method. Methods 25: 402-408, 2001.

11. Li Y, Kang YS, Dai C, Kiss LP, Wen X and Liu Y: Epithelial-to-mesenchymal transition is a potential pathway leading to podocyte dysfunction and proteinuria. Am J Pathol 172: 299-308, 2008.
12. Thornhill BA, Burt LE, Chen C, Forbes MS and Chevalier RL: Variable chronic partial ureteral obstruction in the neonatal rat: A new model of ureteropelvic junction obstruction. Kidney Int 67: 42-52, 2005.

13. Seseke F, Thelen P, Heuser M,Zöller G and Ringert RH: Impaired nephrogenesis in rats with congenital obstructive uropathy. J Urol 165: 2289-2292, 2001.

14. Yu XX, Wang CL, Sun RG, et al: Renal pathological appearance and podocyte phenotype after the ureteral obstruction. Journal of China Medical University 39: 1-3, 2010

15. Dominguez R and Holmes KC: Actin structure and function. Annu Rev Biophys 40: 169-186, 2011.

16. Small DM, Coombes JS, Bennett N, Johnson DW and Gobe GC Oxidative stress, anti-oxidant therapies and chronic kidney disease. Nephrology (Carlton) 17: 311-321, 2012.

17. Hebert DN and Molinari M: In and out of the ER: Protein folding, quality control, degradation, and related human diseases. Physiol Rev 87: 1377-1408, 2007.

18. Ellgaard L, Molinari M and Helenius A: Setting the standards: Quality control in the secretory pathway. Science 286: 1882-1888, 1999.

19. Dickhout JG, Carlisle RE and Austin RC: Interrelationship between cardiac hypertrophy, heart failure, and chronic kidney disease: Endoplasmic reticulum stress as a mediator of pathogenesis. Circ Res 108: 629-642, 2011.

20. Salahudeen AK, Huang H, Joshi M, Moore NA and Jenkins JK: Involvement of the mitochondrial pathway in cold storage and rewarming-associated apoptosis of human renal proximal tubular cells. Am J Transplant 3: 273-280, 2003

21. Manucha W, Kurbán F, Mazzei L, Benardón ME, Bocanegra V, Tosi MR and Vallés P: eNOS/Hsp70 interaction on rosuvastatin cytoprotective effect in neonatal obstructive nephropathy. Eur J Pharmacol 650: 487-495, 2011.

22. Lodha S, Dani D, Mehta R, Bhaskaran M, Reddy K, Ding G and Singhal PC: Angiotensin II-induced mesangial cell apoptosis: Role of oxidative stress. Mol Med 8: 830-840, 2002.

23. Frerman FE: Acyl-CoA dehydrogenases, electron transfer flavoprotein and electron transfer flavoprotein dehydrogenase. Biochem Soc Trans 16: 416-418, 1988

24. Beckmann JD and Frerman FE: Electron-transfer flavoprotein-ubiquinone oxidoreductase from pig liver: Purification and molecular, redox, and catalytic properties. Biochemistry 24 : 3913-3921, 1985

25. Hirokawa S, Shimanuki T, Kitajima H, Nishimori Y and Shimosaka M: Identification of ETFB as a candidate protein that participates in the mechanoregulation of fibroblast cell number in collagen gel culture. J Dermatol Sci 64: 119-126, 2011.

26. Hirokawa S, Shimanuki T, Kitajima H, Nishimori Y and Shimosaka M: Knockdown of electron transfer flavoprotein $\beta$ subunit reduced TGF- $\beta$-induced $\alpha$-SMA mRNA expression but not COL1A1 in fibroblast-populated three-dimensional collagen gel cultures. J Dermatol Sci 68: 179-186, 2012. 